\title{
Automated Surveillance System and Data Communication
}

\author{
B. Suresh Kumar ${ }^{1}$ H. Venkateswara Reddy ${ }^{2}$ C. Jayachandra ${ }^{3}$ \\ ${ }^{1}$ M.Tech (C.S.E), VCE, Hyderabad, India, \\ ${ }^{2}$ Associate Professor in CSE, VCE, Hyderabad, India, \\ ${ }^{3}$ M.Tech (C.S.E),VCE, Hyderabad, India,
}

\begin{abstract}
Automated video surveillance systems plays vital role in the business areas, military system and etc. Many research areas in video surveillance system is mainly focuses on algorithms to evaluate background subtraction system and alert to the system, to repeatedly detect major actions For example, our proposed technique stores, for each pixel, a set of values taken in the past at the same location or in the neighborhood. It then compares this set to the current pixel value in order to determine whether that pixel belongs to the background, and adapts the model by choosing randomly which values to substitute from the background model. This approach differs from those based upon the classical belief that the oldest values should be replaced first. In background comparison if the difference in the values reaches the threshold value then user can get alert message. So whenever user getting SMS from server system and the background image is updated whenever the system is detecting a motion.

Key words : Video surveillance system, Fuzzy Color Histograms, GSM system.
\end{abstract}

\section{Introduction}

Automated video surveillance is a main important study in the business areas. Technology has meet a position where growing up cameras to capture video descriptions is cheap, but finding obtainable human resources to sit and watch that imagery is costly. Video Surveillance systems are become very normal in business areas. Camera output being stored to tapes or stored in video files. later than a crime occurs example : If a store is robbed or a car is stolen the investigators can go back after the fact to see what happened, but of course by then it is too late. So that here what is required in uninterrupted 24-hour monitoring is study of video surveillance records to organize safety officers or major person for prevent the crime.

Many present research areas in video surveillance system is mainly focuses on algorithms to evaluate video, to repeatedly detect major actions [1]. Example applications include intrusion detection, activity monitoring, and walker counting. The facility of extracting moving objects from a video sequence is a basic and critical crisis in the video surveillance systems. For systems using still cameras, background subtraction is the method normally used to fragment moving regions in the image sequences, by comparing each frame to a model of the scene background [2,3].

\section{A. Video Surveillance Systems}

Video surveillance is most important issue in recent years. Object detection and tracking in video surveillance systems are typically depend on background view and subtraction. Video analysis technology can be useful to extend smart surveillance systems that can be help the human operator in real-time threat detection [4]. Specifically, multi scale detecting methods are the expectations step in applying regular video analysis to surveillance systems. Application of visual surveillance include car and walker traffic monitoring, human movement surveillance for strange activity detection, people counting, etc. functions of a video surveillance system contain three main modules : moving object detection, object tracking and motion analysis.

Many video surveillance products are existing on the market for office and home security as well as remote surveillance for monitoring, and for capturing motion actions using webcams and detect intruders [5]. In case of webcams, the image data is saved into compressed or uncompressed video clips, and the system activate a choice of alerts such as sending an e-mail, MMS or SMS.

\section{B. Video System For Rural Surveillance}

The difficulty of object detection has been solved by using statistical models of the background image $[6,5,8]$, frame differences techniques or a combination of both [7]. Many techniques are adapted for object tracking in video sequences in order to run with several interacting targets. Object recognition is performed by applying geometric Pattern Recognition methods. Several features, which find out the exact condition of the problem, can be used. These contain geometric features such as bounding box aspect ratio, motion patterns and color histogram $[5,8]$.Here in this paper we using the color histograms for finding the objects in the surveillance. 


\section{Detection}

The main difficulty in detection is background can formulate a frequent change, frequently use to the reality of amplification variations and distracters (example: clouds passing by, braches of trees moving with the wind). The toughness towards enlightenment difference of the scene is achieved using adaptive background models and adaptive per-pixel thresholds. The per-pixel threshold is then initialized to be above the difference between the two backgrounds. Incident detection, detecting and tracking objects are a significant facility for surveillance. From the viewpoint of a human analyst, the main serious challenge in video based surveillance is interpreting the automatic analysis data to detect events of interest and identify trends. There are two methods for object detection those are background subtraction and salient motion detection. Background subtraction assumes a stationary background and treats all changes in the scene as objects of interest, while salient motion detection assumed that a scene will have many different types of motion of which some types are of interest from a surveillance perspective.

\section{Tracking}

The intention of tracking is to choose the spatial sequential information of each object present in the scene. Since the image motion of targets is always little in contrast to their spatial extends, no spot calculation is essential to build the strokes [26]. The relationship of regions and their classification is depend on a binary association matrix computed by testing the overlap of regions in repeated frames. Whenever there is a match, the stroke is updated. Tracking also interacts with the detection. When a object stops in the scene for a certain amount of time, the tracker merges the target in the background.

\section{E. Back ground subtraction}

Detection of moving objects in video streams is known to be a significant, and difficult, research problem [8]. Apart from the essential value of being able to section video streams into moving and background mechanism, detecting moving blobs provides a center of attention for recognition, classification, and activity analysis, making these later processes more efficient since only "moving" pixels need be measured.

With increasing interest in high-level safety and security, smart video surveillance systems, which enable advanced operations such as object tracking has been in critical demand. For the success of such systems, background subtraction is one of critical jobs in video surveillance, has been measured in different environments. The basic idea of earlier work for this task is to evaluate the difference of pixel values between the reference and current frames. However, this approach is absolutely sensitive to still small variations since it lacks adaptive updating of the reference background. To cope with this restriction, Stauffer and Grimson [9] formulate the distribution of each pixel value over time as a mixture of Gaussians (MoG), which is adaptively updated in an online manner, and then categorize incoming pixels into either background or not. Inspired by their probability model and online updating scheme, numerous variants have been proposed over the last decade [10]-[11].To efficiently suppress dynamic textures in the background and use the Eigen value-based distance metric to update the background model. On the other hand, saliency detection techniques have been recently employed since those have a great ability to detect visually important regions (i.e., moving objects in video sequences) while competently suppressing unrelated backgrounds [12], [13]. even though these methods supply amazing improvements, they still frequently be unsuccessful to rapidly adapt to a range of background motions. Moreover, some methods (e.g.,[10]]) need really high computational costs to be implemented.

\section{Fuzzy Color Histogram And Its Application To Background Subtraction A. Fuzzy Membership Based Local Histogram Features}

The idea of using FCH in a general way to get the consistent background model in active texture scenes is motivated by the study that background motions do not make severe alterations of the scene structure even though they are widely distributed or occur abruptly in the spatiotemporal domain, and color variations yielded by such irrelevant motions can thus be efficiently attenuated by considering local statistics defined in a fuzzy manner, i.e., regarding the effect of each pixel value to all the color attributes rather than only one matched color in the local region. Therefore, it is thought that fuzzy membership based local histograms cover a way for robust background subtraction in dynamic texture scenes. In this subsection, we summarize the FCH model [14] and examine the properties related to background subtraction in dynamic texture scenes.

Here the probability for pixels in the image to belong to the $i$ th color bin $w_{i}$ can be defined as follows:

$$
h_{i}=\sum_{j=1}^{N} P\left(\frac{w_{i}}{x_{j}}\right) P\left(x_{j}\right)=\frac{1}{N} \sum_{j=1}^{N} P\left(\frac{w_{i}}{x_{j}}\right)
$$


where $N$ denotes the total number of pixels. $\mathrm{P}\left(\mathrm{x}_{\mathrm{j}}\right)$ is the probability of color features selected from a given image being those of the $j$ th pixel, which is determined as $1 / N$. The conditional probability $\mathrm{P}\left(\mathrm{w}_{\mathrm{i}} / \mathrm{x}_{\mathrm{j}}\right)$ is 1 if the color feature of the selected $j$ th pixel is quantized into the $i$ th color bin and 0 otherwise. Since the quantized color feature is assumed to be fallen into exactly one color bin, it may lead to the abrupt change even though color variations are actually small. In contrast to that, FCH utilizes the fuzzy membership [15] to relax such a strict condition. More specifically, the conditional probability $\mathrm{P}\left(\mathrm{w}_{\mathrm{i}} \mid \mathrm{x}_{\mathrm{j}}\right)$ of (1) represents the degree of the belongingness for color features of the $j$ th pixel to the $\mathrm{i}$ th color bin (i.e., fuzzy membership $u_{i j}$ ) in FCH and it thus enables to be robust to the noise interference and quantization error.

Using the FCM clustering system ( $\mathrm{m}>\mathrm{c}$ ). That is, by conducting FCM clustering, we can obtain the membership values of a given pixel to all FCH bins. More specifically, the FCM algorithm finds a minimum of a heuristic global cost function defined as follows [15]:

$$
j=\sum_{i=1}^{c} \sum_{j=1}^{m}\left[\mathrm{P}\left(\frac{w_{i}}{x_{j}}\right)\right]^{b}\left\|x_{j}-v_{i}\right\|^{2}
$$

where $x$ and $v$ denote the feature vector (e.g., values of each color channel) and the cluster center, respectively. $b$ is a constant to control the degree of blending of the different clusters and is generally set to 2 . Then we have following equations, i.e., $\partial J / \partial v_{i}=0$ and $\partial J / \partial P_{j}=0$ where $P_{j}$ denotes the prior probability of $\mathrm{P}\left(\mathrm{w}_{\mathrm{j}}\right)$, at the minimum of the cost function. These lead to the solution given as

$$
\begin{gathered}
v_{i}=\frac{\sum_{j=1}^{m}\left[\mathrm{P}\left(\frac{w_{i}}{x_{j}}\right)\right]^{b} x_{j}}{\sum_{j=1}^{m}\left[\mathrm{P}\left(\frac{w_{i}}{x_{j}}\right)\right]^{b}} \\
P\left(w_{i} \mid x_{j}\right)=u_{i j}=\frac{\left(\frac{1}{d_{i j}}\right)^{1 /(b-1)}}{\sum_{r=1}^{c}\left(\frac{1}{d_{r j}}\right)^{1 /(b-1)}}
\end{gathered}
$$

where $\mathrm{d}_{\mathrm{ij}}=\left\|\mathrm{x}_{\mathrm{j}}-\mathrm{v}_{\mathrm{i}}\right\|^{2}$. Since (3) and (4) rarely have logical solutions, these (i.e., cluster center and membership value) are expected iteratively according to [15]. It is worth noting that these membership values derived from (4) only need to be computed once and stored as a membership matrix in advance. Therefore, we can simply build FCH for the incoming video frame by directly referring to the stored matrix without computing membership values for each pixel. For the robust background subtraction in dynamic texture scenes, we finally define the local FCH feature vector at the $j$ th pixel position of the $k$ th video frame as follows:

$$
F_{j}(k)=\left(f_{j, 1}^{k}, f_{j, 2}^{k}, f_{3,1}^{k}, \ldots \ldots \ldots f_{j, \mathrm{c}}^{k}\right), \quad f_{j, 1}^{k}=\sum_{q \in w_{j}^{k}} u_{i q}
$$

where $w_{j}^{k}$ denotes the set of adjacent pixels centered at the position $j . u_{i q}$ denotes the membership value obtained from (4), representing the belongingness of the color feature computed at the pixel position $q$ to the color bin $i$ as mentioned. By using the difference of our local features defined in (5) between successive frames, we can build the reliable background model.

FCH provides pretty reliable outcome even still dynamic textures are commonly spread in the background. Therefore, it is thought that our local FCH features are very useful for modeling the background in dynamic texture scenes. In the following, we will explain the updating scheme for background subtraction based on the similarity measure of local FCH features.

B. Background Subtraction with Local FCH Features

In this part, we explain the process of background subtraction based on our local FCH features. To classify a given pixel into either background or moving objects in the current frame, we first compare the observed FCH vector with the model FCH vector renewed by the online update as expressed in (6): 


$$
B_{j}(k)=\left\{\begin{array}{l}
1, \operatorname{ifS}\left(\mathrm{F}_{j}(k), F_{j}(k)\right)>\tau \\
0, \text { otherwise }
\end{array}\right.
$$

where $B_{j}(k)=1$ denotes that the $j$ th pixel in the $k$ th video frame is determined as the background whereas the corresponding pixel belongs to moving objects if $\mathrm{B}_{\mathrm{j}}(\mathrm{k})=0 . \tau$ is a threshold value ranging from 0 to 1 . The similarity measure S(...) used in (6), which adopts normalized histogram connection for simple calculation, is defined as follows:

$$
S\left(F_{j}(k), F_{j}(k)\right)=\frac{\sum_{i=1}^{c} \min \left[\mathrm{f}_{j, 1}^{k}, \mathrm{f}_{j, 1}^{k}\right]}{\max \left[\sum_{i=1}^{c} \mathrm{f}_{j, 1}^{k}, \sum_{i=1}^{c} \mathrm{f}_{j, 1}^{k}\right]}
$$

where $F_{j}(k)$ denotes the background model of the $j$ th pixel position in the $k$ th video frame, defined in (8). Note that any other metric (e.g., cosine similarity, Chi-square, etc.) can be employed for this similarity measure without significant performance drop. In order to maintain the reliable background model in dynamic texture scenes, we need to update it at each pixel position in an online manner as follows:

$$
F_{j}(\mathrm{k})=(1-\alpha) \cdot F_{j}(k-1)+\alpha \cdot F_{j}(k), \quad \mathrm{k} \geq 1
$$

where $F_{j}(0)=F_{j}(0) . \alpha \in[0,1]$ is the learning rate. Note that the larger denotes that local FCH features currently observed strongly affect to build the background model. By doing this, the background model is adaptively updated. For the sake of completeness, the main steps of the proposed method is summarized in Algorithm 1.

\begin{tabular}{|l|l|}
\hline \multicolumn{2}{|l|}{ Algorithm 1 Background subtraction using local FCH features } \\
\hline Step 1. & $\begin{array}{l}\text { Construct a membership matrix using fuzzy -means } \\
\text { clustering based on (3) and (4) (conducted offline only } \\
\text { once). }\end{array}$ \\
\hline Step 2. & $\begin{array}{l}\text { Quantize RGB colors of each pixel at the } k \text { th video frame } \\
\text { into one of } m \text { histogram bins (e.g., } r \text { th bin where } \\
\mathrm{r}=1,2,3, \ldots \ldots . \mathrm{m} \text { ). }\end{array}$ \\
\hline Step 3. & Find the membership value $\mathrm{u}_{\mathrm{ir}}$ at each pixel position \\
\hline Step 4. & $\begin{array}{l}\text { Compute local FCH features using (5) at each pixel } \\
\text { position of the } k \text { th video frame. }\end{array}$ \\
\hline Step 5. & Classify each pixel into background or not based on (6) \\
\hline Step 6. & Update the background model using (8). \\
\hline Step 7. & Go back to Step 2 until the input is terminated $(\mathrm{k}=\mathrm{k}+1)$ \\
\hline
\end{tabular}

\section{Proposed System}

The purpose of video surveillance systems is to monitor the activity in a specified, indoor or outdoor area. Because the image is usually captured by a stationary camera, it is easier to detect a still background than moving object. Since the cameras used in surveillance are typically stationary, a straightforward way to detect moving objects is to compare each new frame with a reference frame, representing in the best possible way the scene background. The background subtraction is the higher level processing modules for object tracking, event detection and scene understanding purposes uses the results of this process. Successful background subtraction plays a key role in obtaining reliable results in the higher level processing tasks. Background modeling is commonly carried out at pixel level. At each pixel, a set of pixel features, collected in a number of frames, is used to build an appropriate model of the local background. The figure 1 shows the working sketch of the proposed system. Here in the proposed system we will have images from camera. In this proposed system we adopt the background subtraction algorithm on these images. To recognition moving objects on the background, the basic idea in the background subtraction algorithm is comparing the each pixel value in the two images. . According to the result of moving object detection research on video sequences, the movement of the people is tracked using video surveillance. 


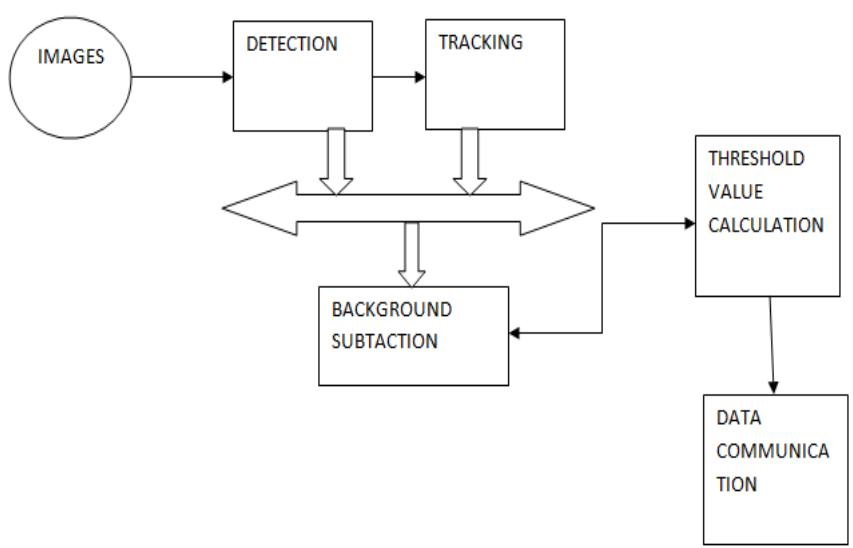

Fig 1.System Block Diagram

The moving object is identified using the image subtraction method. The background image is subtracted from the foreground image. From that the moving object is derived. So the background subtraction algorithm and the threshold value is calculated to find the moving image. Using this algorithm the moving frame is identified. Then by the threshold value the movement of the frame is identified and tracked. Hence the movement of the object is identified accurately.

When the threshold value reached at particular value $\theta$ them the user can get the SMS through the Global System for mobile communication (GSM) system .The GSM system is present in the data communication.

\section{Experimental Results}

In this section, we demonstrate the performance of the proposed work on automated video surveillance system thorough experimental study on the real dataset. In Section VII.I, the test environment and the dataset used are described and finally the evolving processes of surveillance results are visualized on the real dataset.

A. Test Environment and Dataset. All of our experiments are conducted on a PC with an Intel Corei3 processor with $1 \mathrm{~GB}$ memory and the Windows xp operating system. In all experiments, the background subtraction algorithm is chosen todo the object detection on the datasets. For to detecting the object in images we adopt a fuzzy color histograms method to this system. For to developing this paper we use the Dot Net frame work and backend as My Sql .

Here we are developing this project on real time data, that by using Web -camera we capture the images . Then we implement the our source code (Developed based on the $\mathrm{FCH}$ ) on that images, by using that algorithm every image is compared with another neighboring image. In that each comparison it will calculate the threshold value, if the lot of changes accord in the pixel comparison then the proposed system will send a SMS to the user.

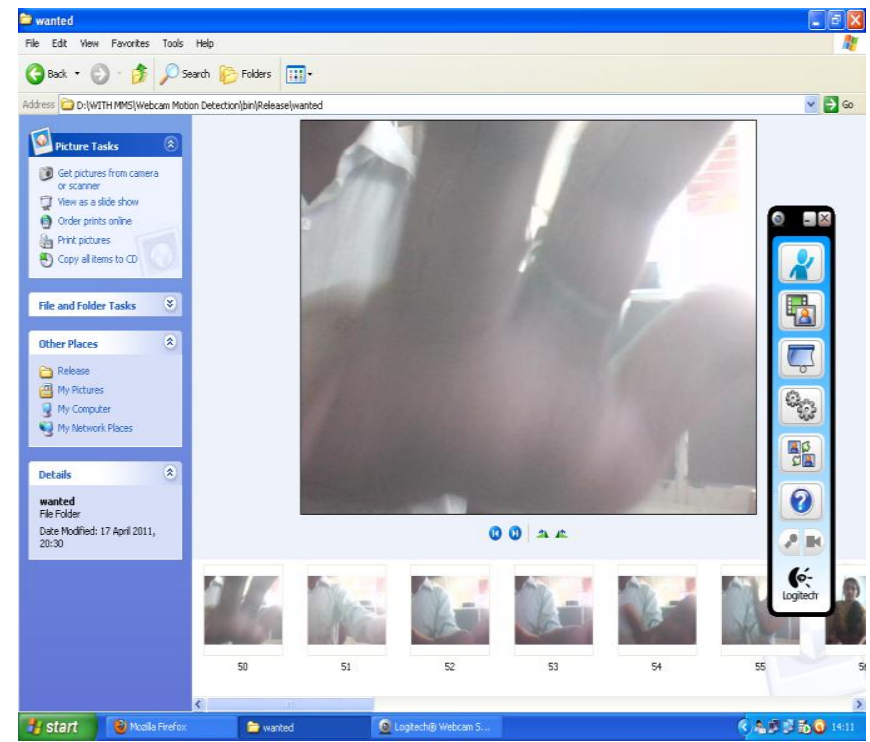

Fig 2: Captured images from the web cam 
The figure 2 shows images that taken from web camera. With using of these captured images we are going execute the proposed system.

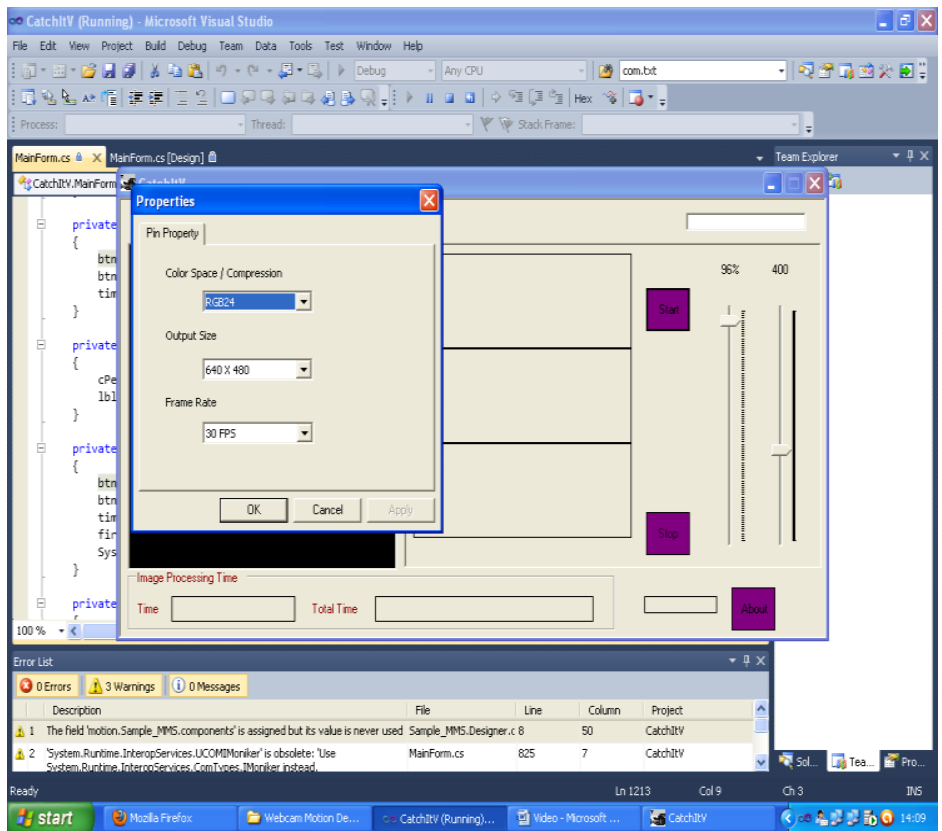

Fig 3: image retrieving information

Fig 3 shows retrieving of images from the specified location, in this stage we are going to give the size of the image and color space and frame rate. After completion of this process the next process that comparison of images. When we complete the previous process then proposed structure will movies to the next step that image processing. In this image processing system the images the images that are stored in the location can be taken into the processing system. That is shown in below Fig 4.

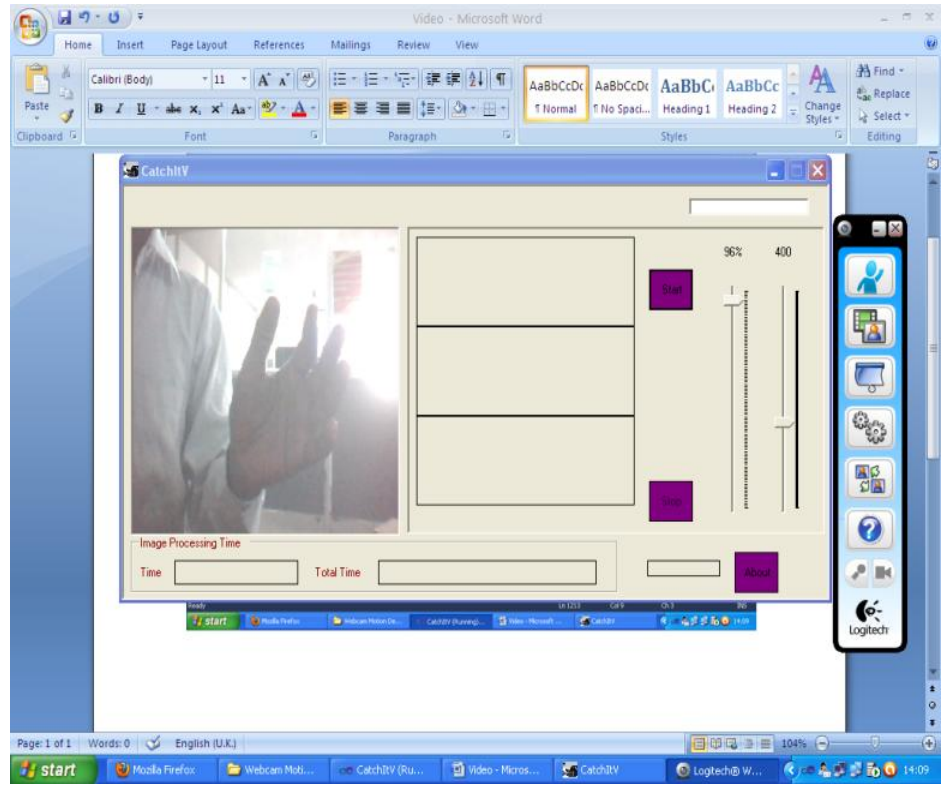

Fig 4: Image processing into the system

By considering these images as input we done background subtraction algorithm. By applying this algorithm on those continuous images we can get the different between the images. That is shown in the below figure 5 . 


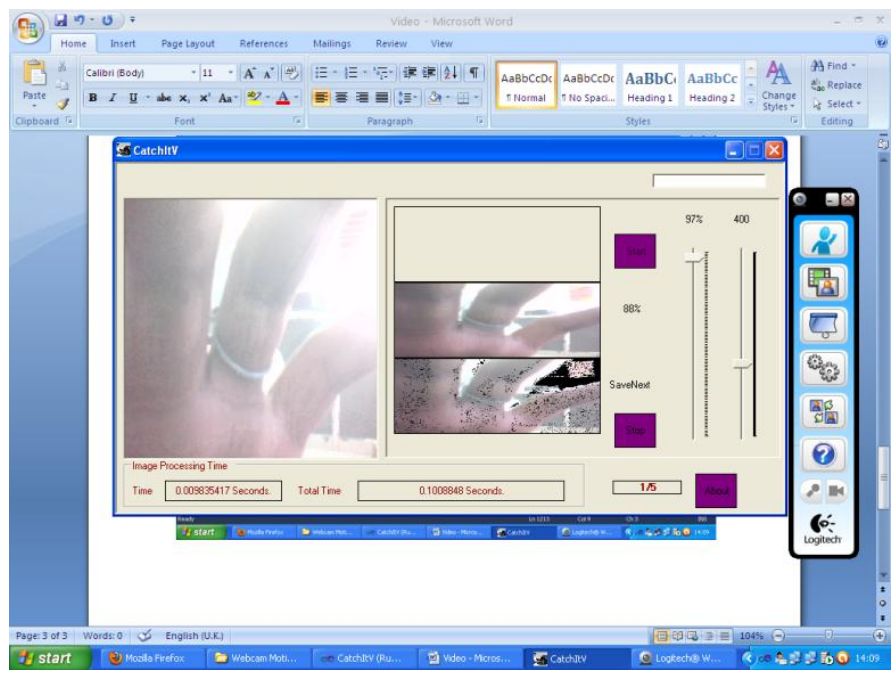

Fig 5: background subtraction of the images

By that comparison we can get different between these two images .when comparison is done at each comparison we get some threshold value, if that threshold value is less we can conclude that there is not a lot changes in the system. If the threshold value is more then we say that there is chance of object or intruder enter in the system. So by using GSM system user can get the SMS alert.

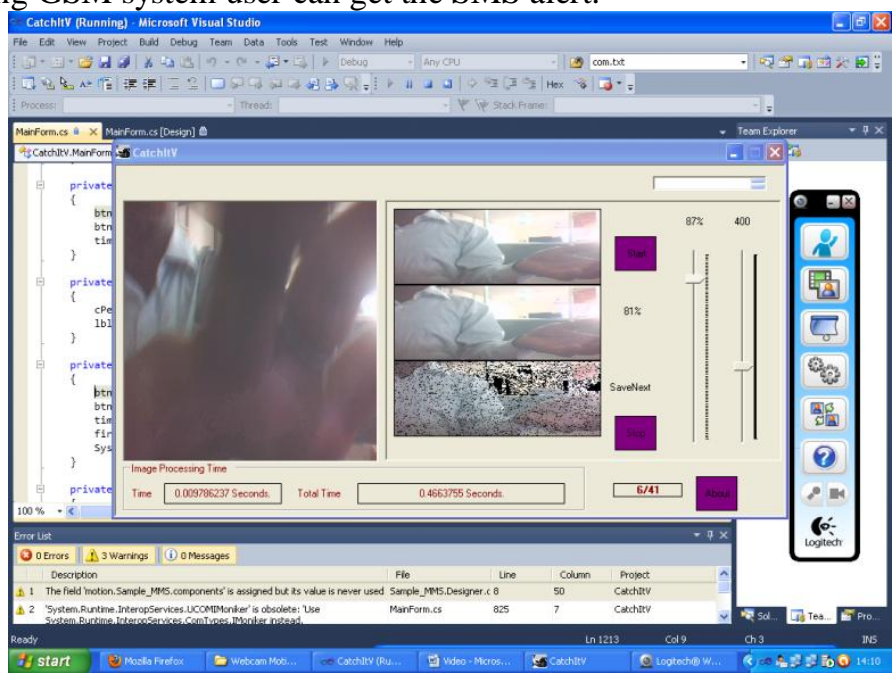

Fig 6 :Threshold Value calculation

With that SMS alert we can get information about what happened in the particular location.

\section{Conclusion}

A technology of background subtraction for real time monitoring system was proposed in this paper. The obvious keystone of my work is studying the principle of the background subtraction, discussing the problem of the background, and exploring the base resolve method of the problem. Experiment shows that the method has good performance and efficiency. When the background subtraction level reach at threshold $\mathrm{v}$ alert the user can get alert message sending multimedia SMS by using GSM (global system for mobile communication) Modem. So then it is very efficiently find out unauthorized person. The future work for this system is when the user get information or alert message then the user can check that images by using some url system.

\section{References}

[1]. CUCCHIARA, R.: "Multimedia Surveillance systems", Proc. ACM Workshop on Video Surveillance and Sensor Networks, pp. $3-10,2005$.

[2]. ElgAMMAL, A. - HARWOOD, D. - DAVIS, L. A.: "Non-Parametric Model for Background Subtraction", ICCV'99, 1999

[3]. HORPRASERT, T. - HARWOOD, D. - DAVIS, L. A.: "A Statistical Approach for Real-Time Robust Background Subtraction and Shadow Detection", ICCV'99 Frame Rate Workshop, 1999.

[4]. $\quad$ ARAKI, A. - MATSUOKA, T. - YOKOYA, N. - TAKEMURA, H.: "Real-Time Tracking of Multiple Moving Object Contours in a Moving Camera Image Sequence”, IEICE Trans. Inf. \&Syst., vol. E83-D, no. 7, pp. 1583-1591, July 2001.

[5]. HARITAOGLU, H.: "Hartwood and Devis, W4: Real Time Surveillance of People and their Activities", IEEE Trans. Pattern Anal. Machine Intell., vol. 22, no. 8, pp. 809-830, Aug. 2000. 
[6]. BOULT, T. et al.: "Into the Woods: Visual Surveillance of Noncooperative and Camouflaged Targets in Complex Outdoor Settings", in Procceding of the IEEE, vol. 89, no. 10, Oct. 2001.

[7]. COLLINS, R. - et al.: “A System for Video Surveillance and Monitoring”, CMU-RI-TR-00-12, 2000

[8]. $\quad$ McKENNA, S. et al.: "Tracking Groups of People”, CVIU 80, pp. 42-56, 2000

[9]. C. Stauffer and W. E. L. Grimson, "Adaptive background mixture models for real-time tracking," in Proc. IEEE Computer Vision and Pattern Recognition (CVPR), Jun. 1999, vol. 2, pp. 246-252.

[10]. H. Wang and D. Suter, "A re-evaluation of mixture of Gaussian background modeling," in Proc. IEEE Int. Conf. Accoustics, Speech, and Signal Processing (ICASSP), Mar. 2005, vol. 2, pp. 1017-1020.

[11]. B. Zhong, S. Liu, H. Yao, and B. Zhang, "Multi-resolution background subtraction for dynamic scenes," in Proc. IEEE Int. Conf. Image Processing (ICIP), Nov. 2009, pp. 3193-3196.

[12]. S. Zhang, H. Yao, S. Liu, X. Chen, and W. Gao, "A covariance-based method for dynamic background subtraction," in Proc. IEEE Int. Conf. Pattern Recognition (ICPR), Dec. 2008, pp. 1-4.

[13]. C.Guo and L. Zhang, "Anovelmultiresolution spatiotemporal saliency detection model and its applications in image and video compression," IEEE Trans. Image Process., vol. 19, no. 1, pp. 185-198, Jan. 2010.

[14]. V. Mahadevan and N. Vasconcelos, "Spatiotemporal saliency in dynamic scenes," IEEE Trans. Patt. Anal. Mach. Intell., vol. 32, no. 1, pp. 171-177, Jan. 2010 .

[15]. P. Noriega and O. Bernier, "Real time illumination invariant background subtraction using local kernel histograms," in Proc. Brit. Machine Vision Conf. (BMVC), 2006, vol. 3, pp. 1-10.

[16]. J. Han and K.-K.Ma, "Fuzzy color histogram and its use in color image retrieval," IEEE Trans. Image Process., vol. 11, no. 8, pp. 944-952, Nov. 2002.

[17]. $\quad$ R. O. Duda, P. E. Hart, and D. G. Stork, Pattern Classification, $2^{\text {nd }}$ ed. New York: Wiley-Interscience, 2001.

[18]. L. Li, W. Huang, I. Y.-H. Gu, and Q. Tian, "Statistical modeling of complex backgrounds for foreground object detection," IEEE Trans.Image Process., vol. 13, no. 11, pp. 1459-1472, Nov. 2004. 